\title{
Juvenile Rheumatoid Arthritis
}

National Cancer Institute

\section{Source}

National Cancer Institute. Juvenile Rheumatoid Arthritis. NCI Thesaurus. Code C27179.

An older, deprecated term that encompassed three major types of autoimmune or autoinflammatory arthritis in children: systemic-onset, pauciarticular, or polyarticular arthritis. The juvenile rheumatoid arthritis classification system has been replaced by the International League of Associations for Rheumatology (ILAR) juvenile idiopathic arthritis classification system. 